\title{
The kinase activity of PKR represses inflammasome activity
}

\author{
Howard CH Yim ${ }^{1,3}$, Die Wang ${ }^{1,3}$, Liang Yu$^{2,3}$, Christine L White ${ }^{1,3}$, Pieter W Faber ${ }^{4}$, Bryan RG Williams ${ }^{1,3}$, \\ Anthony J Sadler ${ }^{1,3}$ \\ ${ }^{I}$ Centre for Cancer Research, Hudson Institute of Medical Research, 27-31 Wright St, Clayton, Victoria 3168, Australia; ${ }^{2}$ Centre \\ for Innate Immunity and Infectious Diseases, Hudson Institute of Medical Research, 27-31 Wright St, Clayton, Victoria 3168, Aus- \\ tralia; ${ }^{3}$ Department of Molecular and Translational Science, Monash University, Clayton, Victoria 3168, Australia; ${ }^{4} \mathrm{Chicago}$ Ge- \\ nomics Facility, University of Chicago, IL 60637, USA
}

The protein kinase $R(P K R)$ functions in the antiviral response by controlling protein translation and inflammatory cell signaling pathways. We generated a transgenic, knock-in mouse in which the endogenous PKR is expressed with a point mutation that ablates its kinase activity. This novel animal allows us to probe the kinase-dependent and -independent functions of PKR. We used this animal together with a previously generated transgenic mouse that is ablated for PKR expression to determine the role of PKR in regulating the activity of the cryopyrin inflammasome. Our data demonstrate that, in contradiction to earlier reports, PKR represses cryopyrin inflammasome activity. We demonstrate that this control is mediated through the established function of PKR to inhibit protein translation of constituents of the inflammasome to prevent initial priming during innate immune signaling. These findings identify an important role for PKR to dampen inflammation during the innate immune response and caution against the previously proposed therapeutic strategy to inhibit PKR to treat inflammation.

Keywords: inflammation; inflammasomes; protein kinase R; kinase activity; protein translation

Cell Research (2016) 26:367-379. doi:10.1038/cr.2016.11; published online 22 January 2016

\section{Introduction}

An important mechanism by which eukaryotes respond to stress is to modulate protein translation via regulation of the activity of the eukaryotic initiation factor $2 \alpha(\mathrm{eIF} 2 \alpha)[1]$. Repression of eIF2 $\alpha$ activity reprograms translation and transcription to resolve stress in a coordinated response termed the integrated stress response [2]. In humans, the activity of eIF $2 \alpha$ is controlled through inhibitory phosphorylation by four related kinases that respond to separate stress stimuli. One of these kinases, the protein kinase R (PKR, encoded by the eIF2ak2 gene), constitutes part of the innate immune response. PKR has been reported to promote the activity of the cryopyrin (encoded by the NOD-like receptor family, pyrin domain containing 3 (Nlrp3) gene) inflammasome

Correspondence: Anthony J Sadler

Tel: +61 38572 2722; Fax: +61 395947167

E-mail: anthony.sadler@hudson.org.au

Received 25 June 2015; revised 24 October 2015; accepted 4 November

2015; published online 22 January 2016
[3], a multi-protein complex that activates inflammatory caspases (CASP). This proposition appears at odds with the corollary of the integrated stress response, to inhibit general gene expression and relieve stress. It also runs counter to the finding that the cognate interferon response, which induces the expression of PKR, represses inflammasome activity [4]. Two subsequent independent studies have either contradicted [5] or supported [6] the initial report that PKR promotes the activity of the cryopyrin inflammasome. The predominance of the inflammasomes in inflammatory pathology has prioritized efforts to understand how they are regulated in order to appropriately target their activity to mitigate diseases.

The inflammasomes are activated in two steps: first, a priming stimulus induces the expression of sensor proteins and the pro-form of the caspase substrate, interleukin (IL)- $1 \beta$, before a second stimulus triggers assembly of the multi-protein inflammasome complex to activate the pro-CASP-1 zymogen and subsequently process the pro-forms of IL-1 $\beta$ and IL-18. These processed cytokines then signal via their cognate receptors to directly induce inflammation or secondary inflammatory cytokines and 
chemokines, induce pathogenic cytokines and promote recruitment of immune cells. It has been asserted that PKR promotes the activity of the cryopyrin inflammasome by acting as a scaffold molecule [3]. Previous reports have advanced that PKR modulates other cell signaling pathways by acting as a scaffold molecule $[7$, 8]. This activity of PKR appears not to require kinase activity $[9,10]$ and is at least partly mediated through an association with the tumor necrosis factor (TNF) receptor-associated factor (TRAF) adaptor molecules [11]. However, the TRAF adaptors, which have an uncertain role in inflammasome activity [12], were not implicated. Rather, it is contended that PKR directly links the constituents of the inflammasome, and that this function depends on the kinase activity of PKR [3].

To clarify the role of PKR in inflammasome activity, we generated a novel knock-in kinase-dead PKR mouse. We used macrophages from this animal in parallel with an existing PKR-ablated transgenic animal to establish the kinase-dependent and -independent functions of PKR in controlling the activity of the cryopyrin inflammasome.

\section{Results}

\section{Generation of a kinase-dead PKR transgenic mouse}

To dissect the consequences of substrate phosphorylation from other functions of PKR, we generated a kinase-dead transgenic animal by point mutagenesis. Alignment of kinase protein sequences distinguishes an invariant lysine residue within the N-lobe of the kinase domain, within a region termed the Hank's conserved region II [13] that is essential for kinase activity (Figure 1A). This conserved lysine is located within the catalytic pocket of the enzyme and catalyzes the transfer of the phosphate from ATP to the substrate. Mutation of this residue (number 296) to an arginine in human PKR ablates phosphorylation activity [14]. We engineered the equivalent mutation of the lysine residue (number 271) in the murine PKR (Figure 1B). This mutation was introduced into $129 / \mathrm{Sv}$ embryonic stem cells by homologous recombination of an $8240 \mathrm{bp}$ fragment of the eIF2ak2 locus that encodes two altered nucleotides. A floxed neomycin gene divided this fragment into two arms that centred on intron 9, so that CRE-mediated excision produced a $\operatorname{lox} P$ site within intron 9 and a changed codon (AAG > CGG) in exon 10 at nucleotide positions 811813 within the open reading frame of the gene. This change altered the critical phosphotransfer site lysine 271 to an arginine (K271R) (Figure 1B). Correct incorporation of the targeting cassette into the genome of neomycin-resistant clones was determined by Southern blot using DNA probes that detect sequence immediately $5^{\prime}$ and $3^{\prime}$ to the targeting cassette, within introns 4 and 12 of the genetic locus. Endonuclease digestion and southern hybridization distinguished the wild-type $13010 \mathrm{bp} \mathrm{Pa}$ $c \mathrm{I}-X h o \mathrm{I}$ and $7171 \mathrm{bp}$ EcoRV-XhoI fragments from 7720 bp PacI-PacI and $5273 \mathrm{bp} X h o \mathrm{I}-X h o \mathrm{I}$ fragments in the recombinant genome, produced by additional restriction endonuclease sites ( $\mathrm{PaCI}$ and $\mathrm{XhoI}$ ) within the targeting cassette (depicted in Figure 1B). Subsequent excision of the floxed neomycin cassette was confirmed by two separate PCR reactions. The first amplified a product within the neomycin cassette that is lost by CRE-mediated excision. The second, priming from within intron 9 and exon 10 of the gene locus, amplifies sequence that encompass the floxed neomycin cassette. In this instance, three different amplicons are produced that distinguish the wildtype sequence, full-length targeted construct (containing the floxed neomycin cassette) and the CRE-excised construct (containing a flox site within intron 9) (Figure 1B and $1 \mathrm{C})$.

Transgenic cells were introduced into blastocyst and chimeric mice were produced. Correct genomic transfer was confirmed by PCR and the heterozygous mutant animals were backcrossed to the C57B16J mouse for 10 generations. The point mutation was confirmed in the homozygous mutant (K271R), isogenic C57B16J mouse (designated $e I F 2 \alpha k^{\text {tmILri }}$ ) by PCR (Figure 2A) and then sequencing of the eIF2 $\alpha \mathrm{k} 2$ locus (Figure 2B). The loss of kinase activity was verified in fibroblasts from embryos isolated from wild-type, PKR-ablated [15] $\left(e I F 2 \alpha k 2^{-/-}\right)$ and K271R-PKR (eIF2 $\left.\alpha k 2^{t m I L r i}\right)$ mice treated with the PKR-activating ligand polyinosinic:polycytidylic acid (polyI:C) by measuring phosphorylation of eIF $2 \alpha$ and autophosphorylation of PKR by western blot (Figure $2 \mathrm{C})$.

PKR represses production of the inflammatory $I L-1 \beta$ and IL-18 cytokines

We tested the role of PKR in inflammasome activation by measuring the production of IL- $1 \beta$ and IL18 in primary macrophages isolated from wild-type, $e I F 2 \alpha k 2^{-/}$and the kinase-dead K271R-PKR (eIF2 $\alpha k$ $\left.2^{t m I L r i}\right)$ mice. Peritoneal macrophages were treated with lipopolysaccharide (LPS) to activate PKR $[7,16]$ and prime the inflammasome. Because different stimuli that trigger the cryopyrin inflammasome are taken up by cells through distinct mechanisms with differing dependence on the actin cytoskeleton, which is modulated by PKR [17], we used the ionophore nigericin to minimize this potential source of variance [18]. Measures of IL-1 $\beta$ and IL-18 by ELISA demonstrated elevated levels of these cytokines in the supernatant from macrophages isolated 
A

\begin{aligned} & \multicolumn{1}{c}{271} \\ Mm-PKR & DGKRYAIKRVKY \\ & 296 \\ Hs-PKR & DGKTYVIKRVKY \\ Hs-GCN2 & DGCCYAVKRIPINPASRQ \\ Hs-PERK & DDCNYAIKRIRLPNREL \\ Hs-HRI & DGQYYAIKKILIKGATKT \end{aligned}

B

WT eif2ak2 locus $\quad$ K
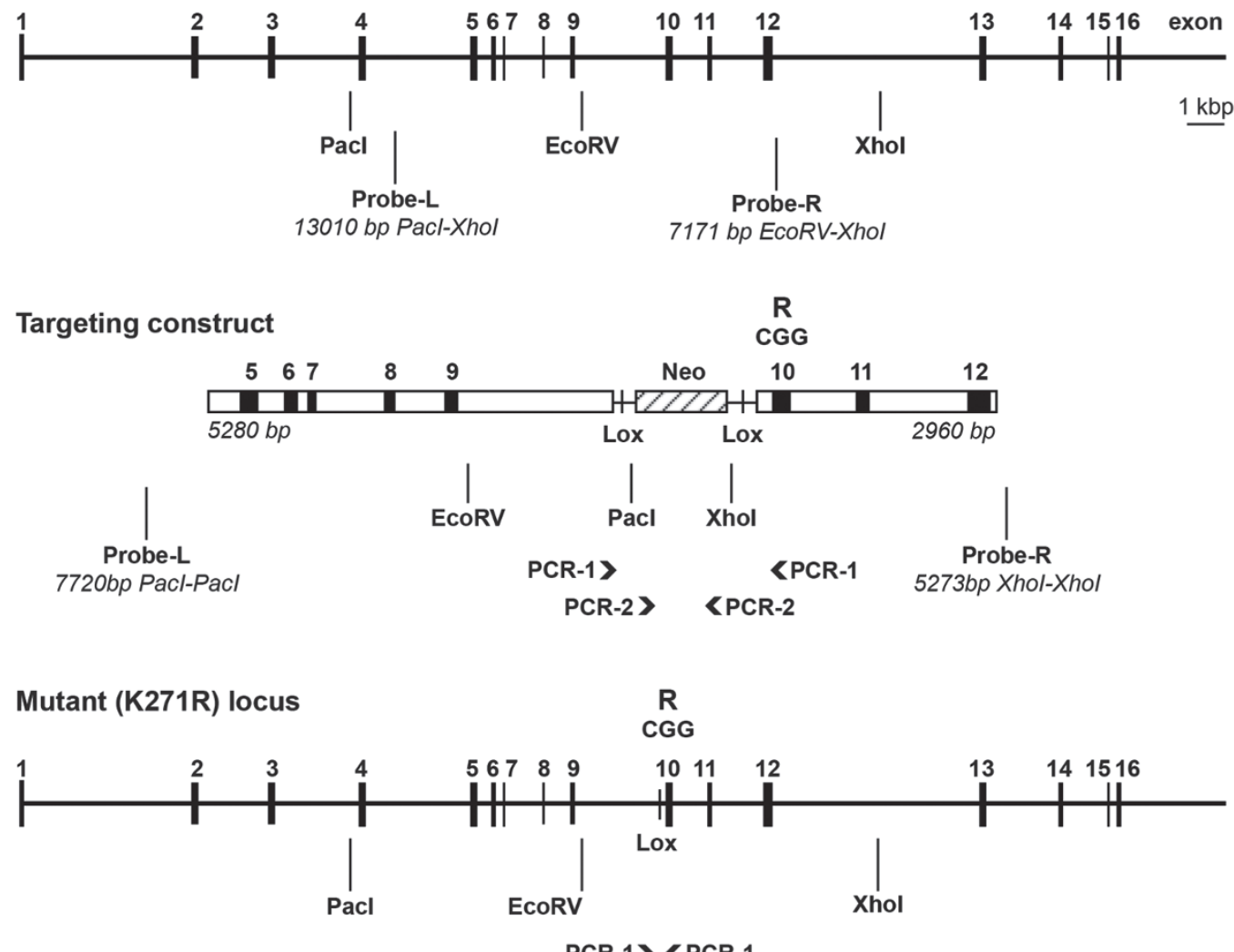

PCR-1 > PCR-1

C

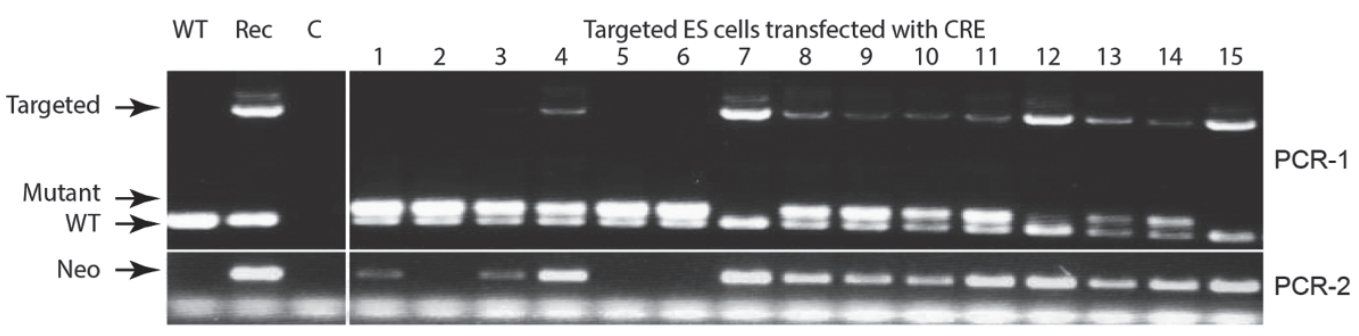

Figure 1 Generation of PKR kinase-dead (K271R) embryonic stem cells. (A) Aligned protein sequence of the Hank's conserved domains of murine PKR (Mm-PKR) with the four human (Hs-) elF2 $\alpha$ kinases, with the essential conserved lysine indicated in bold at position 271 in the murine and 296 in the human PKR protein. (B) A schematic of the wild-type (WT), targeting mutagenic cassette and resulting mutant eif2ak2 gene locus. Restriction endonuclease sites ( $P$ acl, EcoRV and Xhol), position of hybridization probes (Probe-L, Probe-R) and sites of PCR primers (PCR-1 and -2) used to identify the correctly targeted allele are indicated. (C) Image of amplicons generated from PCR reaction (PCR-1 and -2) that were used to distinguish WT, targeted (Rec) and CRE-mediated excision of the mutagenic cassette in embryonic stem cells, resolved by agarose electrophoresis and visualized with SYBR Safe. 
A

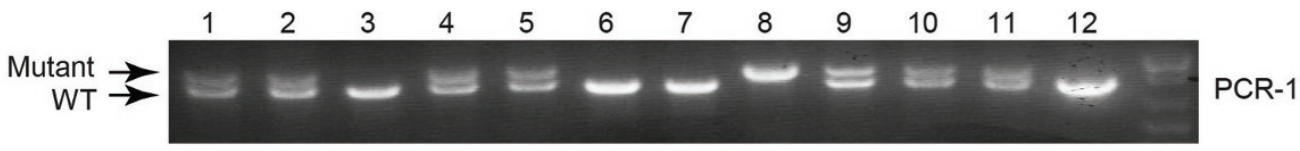

B

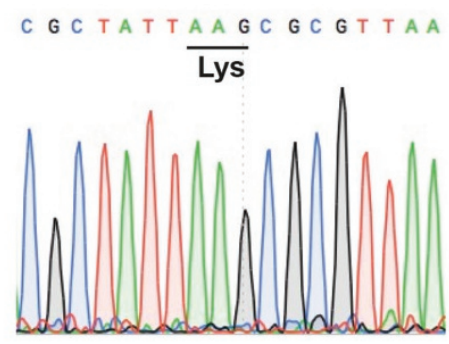

C GCTATTCGGCGCGTTAA

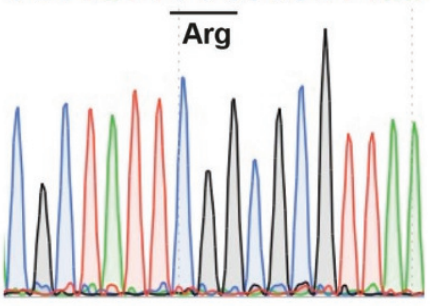

C

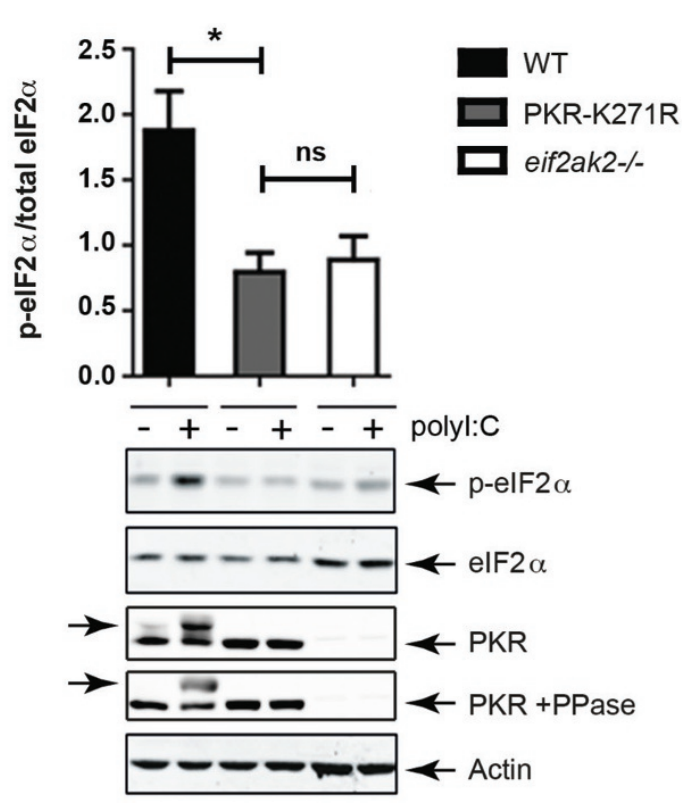

Figure 2 Generation of PKR kinase-dead mice. (A) Amplicons generated by PCR reaction (PCR-1) from the progeny of transgenic PKR-K271R heterozygous mating, resolved by agarose electrophoresis and visualized with SYBR Safe. (B) Sequencing tracers detecting point mutation of two adenines to cytosine and guanine at positions 811 and 812 within the open reading frame of the gene, within exon 10 of the eif2ak2 locus of homozygous mutant PKR-K271R mice, which changes the essential lysine 271 to arginine (K271R). (C) Quantitation of PKR kinase activity by detecting elF2 $\alpha$ phosphorylation and

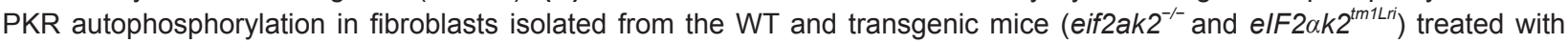
JetPRIME and polyl:C ( $3 \mu \mathrm{g} / \mathrm{ml})$ for $2 \mathrm{~h}$. Whole-cell lysates were probed with anti-pS ${ }^{51}$-elF2 $\alpha$ and -elF2 antibodies $(n=3)$. PKR expression is detected by western blot with an anti-PKR antibody. Autophosphorylation of the protein is detected as an electrophoretic shift in WT compared to the kinase-dead PKR (indicated with arrows), which is reduced by treatment with a protein phosphatase (PPase). An anti-actin antibody is used as an additional loading control. 
from $e I F 2 \alpha k 2^{-/}$and kinase-dead K271R-PKR mice, compared with the wild-type mice (Figure $3 \mathrm{~A}$ and $3 \mathrm{~B}$ ). The anti-IL-18 antibody substantially reacts with the mature cytokine, and thus the levels of IL-18 detected denote inflammasome activity. In addition, equivalence in the levels of the lactate dehydrogenase (LDH) in the cell supernatant of treated cells demonstrates that the heightened levels of IL-1 $\beta$ and IL-18 in cell supernatants with impaired PKR activity was not the result of different release of cytosolic constituents due to variable apoptotic responses (Figure 3C) [19, 20]. Similarly, there was no increase in the release of the CASP-1-independent TNF $\alpha$ in wild-type cells (Figure 3D). Notably, there is increased LPS-dependent induction of TNF $\alpha$ in the K271R-PKR cells compared with the wild-type and PKR-ablated cells (Figure 3D). This is consistent with the previously reported kinase-independent activation of nuclear factor $\kappa \mathrm{B}(\mathrm{NF}-\kappa \mathrm{B})$-dependent cell signaling by PKR [9], and is in accordance with the elevated expression of kinase-dead PKR (Figure 2C) [21]. In keeping with these genetic experiments, treatment of wild-type primary peritoneal macrophages with the PKR inhibitor, 2-aminopurine (2-AP), also promoted production of IL$1 \beta$ as measured by ELISA (Figure 3E). In keeping with the preceding data, concurrent measures of the levels of $\mathrm{TNF} \alpha$ demonstrate that caspase-independent cytokine processing is unaffected by pharmacologically inhibiting PKR (Figure 3F).

These data demonstrate that through its kinase activity PKR diminishes the production of IL- $1 \beta$ and IL-18, suggesting that PKR kinase activity represses inflammasome activity.

\section{PKR represses inflammasome assembly}

A definitive measure of inflammasome assembly is the aggregation of apoptosis-associated speck-like protein containing a CARD (ASC) into a large protein complex in the perinuclear space. Accordingly, we measured the extent of ASC nucleation within PKR mutant or wild-type macrophages treated with LPS and nigericin by immunofluorescence. Consistent with the apparently increased inflammasome activity evidenced by elevated cytokine processing in mutant cells, ablation of PKR expression or kinase activity promoted the aggregation of ASC (Figure 4A). Coalesced ASC directly recruits proCASP-1, via mutual caspase activation and recruitment domain (CARD)-mediated interaction, to activate the protease. Quantitation of CASP-1 activity using fluorochrome-labeled inhibitor of caspases (FLICA) probes in macrophages isolated from wild-type, eIF $2 \alpha 2^{-/-}$and K271R-PKR mice by fluorescence microscopy demonstrated that ablation of PKR expression or kinase activity promoted CASP-1 activity compared with the wild-type macrophages (Figure 4B). In keeping with these measures of ASC nucleation and CASP-1 activity, western blot analysis demonstrated increased cleavage of cytosolic pro-IL-1 $\beta$ in the PKR mutant compared with wildtype macrophages (Figure 4C).

Together, these data demonstrate that the kinase activity of PKR reduces the production of IL- $1 \beta$ and Il-18 by reducing the formation of ASC specks and the subsequent activation of pro-CASP-1.

\section{PKR represses the induction of inflammasome constitu-} ents

To determine the mechanism by which PKR regulates this response, we measured the expression of cryopyrin inflammasome constituents. Analysis of the levels of selected transcripts in wild-type, eIF $2 \alpha \mathrm{k}^{-/-}$and the kinase-dead K271R-PKR macrophages treated with LPS demonstrated that PKR did not alter the transcriptional response (Figure 5A) [20]. However, measures of the levels of gene products demonstrated that PKR repressed the induction of pro-IL-1 $\beta$ and cryopyrin during LPS-dependent priming, although this measure of repression only reached statistical significance for the highly induced pro-IL-1 $\beta$ (wild-type vs $e I F 2 \alpha k 2^{-/-} F=$ 99.6286, $D F n=1, D F d=20, P=0.005607$; wild-type vs PKR-K271R $F=9.89311, D F n=1, D F d=20, P=$ 0.005093 , by linear regression) (Figure 5B and 5C). Notably, this effect was not ubiquitous, as levels of the effector enzyme CASP-1 and critical adaptor protein ASC were equivalent between wild-type and PKR mutant cells (Figure 5B and 5C). This control of the induction of proteins is in keeping with the established function of PKR to inhibit protein translation.

To investigate the significance of translational control for inflammasome activity, we treated wild-type primary peritoneal macrophages with the established protein biosynthesis inhibitor cycloheximide. Equivalent to the preceding experiment, measures of the levels of constituents of the inflammasome by western blot showed that cycloheximide repressed the LPS-dependent induction of pro-IL-1 $\beta$ and cryopyrin, with no major change to the levels of CASP-1 and ASC (Figure 5D). Quantitation of the levels of IL-1 $\beta$ and IL-18 by ELISA demonstrated that the inhibition of protein translation with cycloheximide repressed the activity of the cryopyrin inflammasome (Figure 5E). Notably, the levels of both active cytokines were repressed, although only IL- $1 \beta$ induction is affected. The impairment in the processing of proIL-18 demonstrates that the activity of the cryopyrin inflammasome is controlled by gene expression at the level of protein translation. To verify that PKR imposed 
A

\section{IL-1 $\beta$}

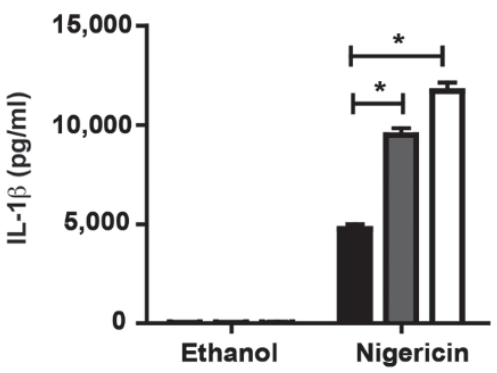

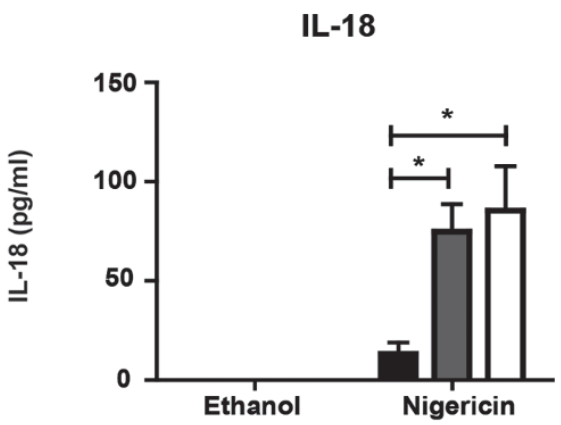

C

LDH

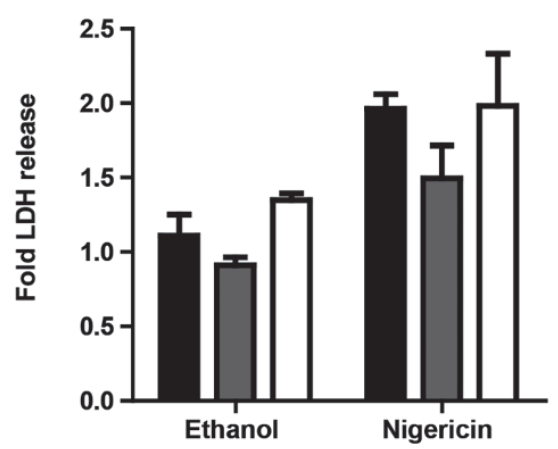

E

\section{IL-1 $\beta$}

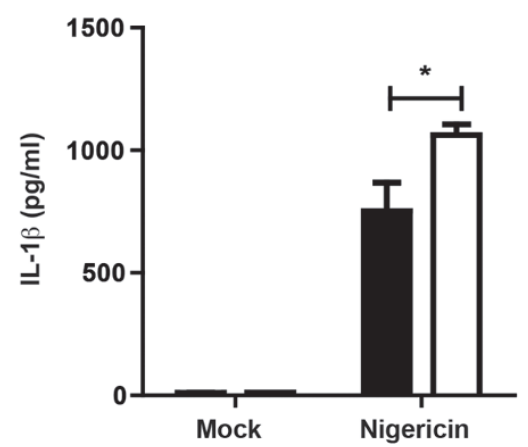

WT

PKR-K271R

eif2ak2-/-

D

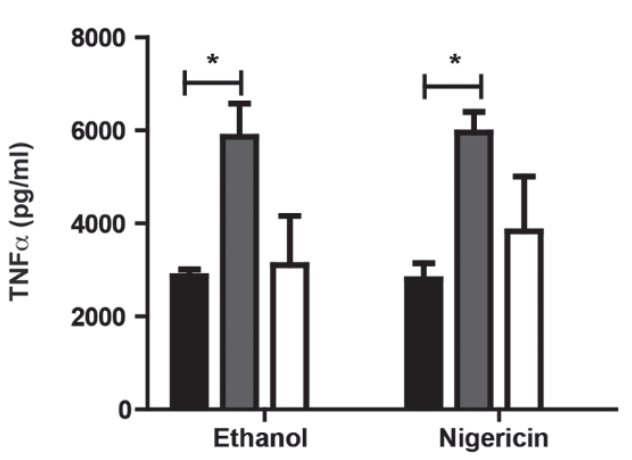

$\mathrm{F}$

TNF $\alpha$

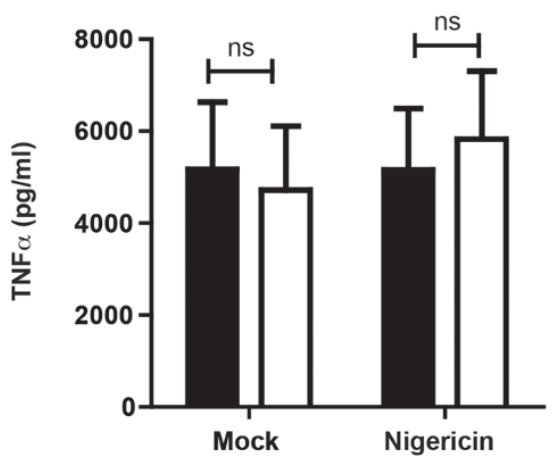

Figure 3 Ablating the expression or kinase activity of PKR suppresses the production of IL-1 3 and IL-18. (A, B) Production of IL-1 $\beta(A)$ and IL-18 (B) in the culture supernatant of peritoneal macrophages from WT and transgenic mice (eif2ak2 ${ }^{-/-}$ and elF2 $\left.\alpha k 2^{t m 1 L r i}, n=3\right)$, primed for $4 \mathrm{~h}$ with LPS $(10 \mathrm{ng} / \mathrm{ml})$ then nigericin $(10 \mu \mathrm{M})$ for $2 \mathrm{~h}$ for IL-1 $1 \beta$, or $30 \mathrm{~min}$ for IL-18. (C) Assessment of membrane integrity by LDH cytotoxicity assay in the culture supernatant of peritoneal macrophages from WT and transgenic mice (eif2ak $2^{-/}$and elF2 $\alpha k 2^{\text {tm1 Lri }}, n=3$ ), primed for $4 \mathrm{~h}$ with LPS (10 ng/ml), then nigericin (10 $\left.\mu \mathrm{M}\right)$ for $30 \mathrm{~min}$. (D) Assessment of TNF $\alpha$ production in the culture supernatant of peritoneal macrophages from WT and transgenic mice (eif2ak2 ${ }^{-/-}$ and elF2 $\left.\alpha k^{t m 1 L r i}, n=3-4\right)$, primed for $4 \mathrm{~h}$ with LPS $(10 \mathrm{ng} / \mathrm{ml})$, then nigericin $(10 \mu \mathrm{M})$ for $2 \mathrm{~h}$. (E, F) Production of IL-1 $\beta$ (E) and TNF $\alpha(F)$ in the culture supernatant of peritoneal macrophages from WT mice $(n=3)$ treated with the PKR inhibitor 2-AP $(1 \mathrm{mM})$ for $1 \mathrm{~h}$ before stimulation with LPS $(10 \mathrm{ng} / \mathrm{ml})$ for $4 \mathrm{~h}$, then nigericin $(10 \mu \mathrm{M})$ for 30 min. Cytokine levels are measured by ELISA $\left({ }^{*} P<0.05\right)$. 
A

\section{ASC nucleation}

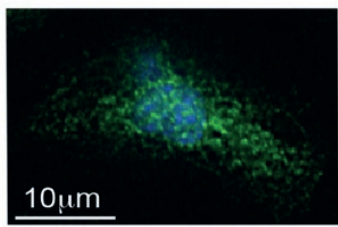

Ethanol

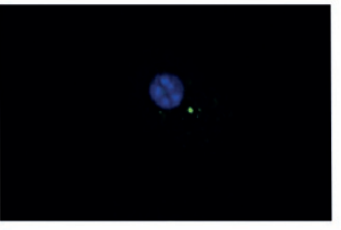

Nigericin

B

CASP-1 activity

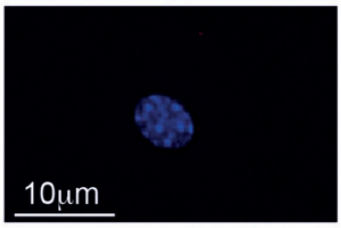

Ethanol

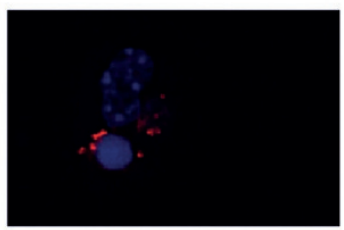

Nigericin
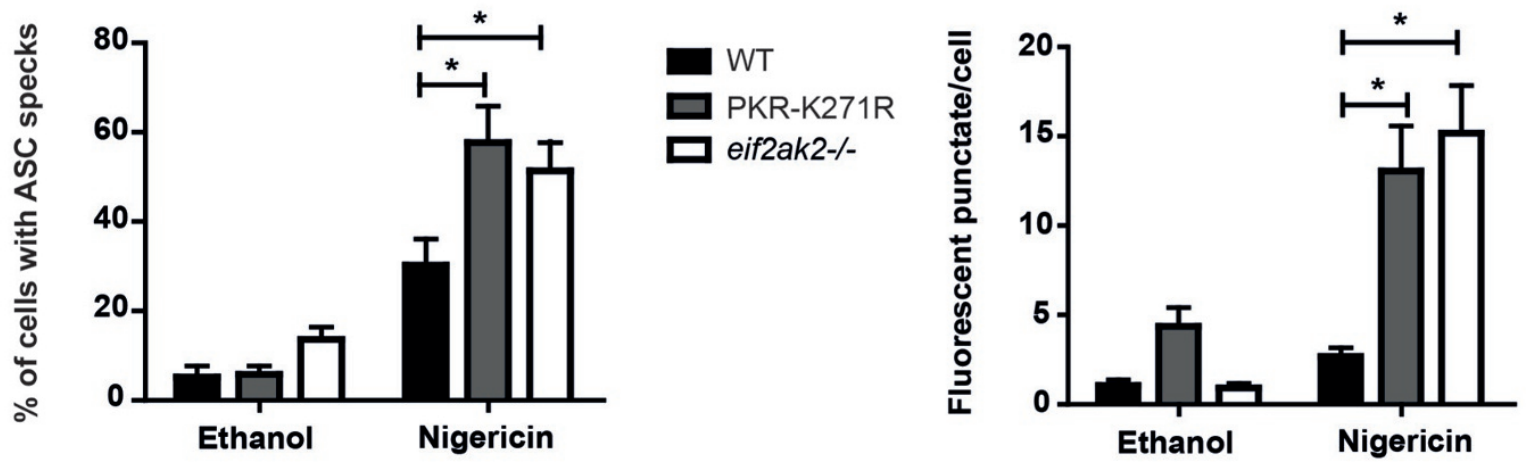

C

\section{Pro-IL-1 $\beta$ clevage}

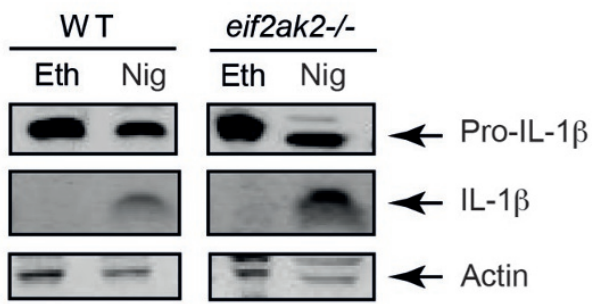

Figure 4 PKR suppresses the assembly of the cryopyrin inflammasome. (A) A micrograph (at top) demonstrating ASC nucleation, which is quantitated in the graph below in peritoneal macrophages from WT and PKR mutant mice $(n=3-4)$ that were treated with LPS $(10 \mathrm{ng} / \mathrm{ml})$ for $4 \mathrm{~h}$, then either the solute (ethanol) or nigericin $(10 \mu \mathrm{M})$ for 15 min. The ASC protein is detected by immunofluorescence with an anti-ASC antibody. (B) A micrograph (at top) demonstrating CASP-1 activity, which is quantitated in the graph below in peritoneal macrophages from WT and PKR mutant mice $(n=3)$ that were treated with LPS $(10 \mathrm{ng} / \mathrm{ml})$ for $4 \mathrm{~h}$, then either the solute (ethanol) or nigericin $(10 \mu \mathrm{M})$ for $15 \mathrm{~min}$. CASP-1 activity is measured using FLICA. ( ${ }^{\star} P$ $<0.05$ ) (C) Immune blot detection of the processing of pro-IL-1 $\beta$ in lysates from WT and eif2ak $2^{-/-}$peritoneal macrophages treated with LPS $(10 \mathrm{ng} / \mathrm{ml})$ for $4 \mathrm{~h}$, then either the solute (Eth) or nigericin (Nig, $10 \mu \mathrm{M})$ for $30 \mathrm{~min}$.

its effects through eIF $2 \alpha$ phosphorylation, we tested an inhibitor of this response pathway for its effect on the expression of inflammasome constituents. In keeping with the preceding data, pre-treatment of peritoneal macrophages with the small molecule integrated stress response inhibitor, ISRIB, which counteracts the effect of eIF $2 \alpha$ phosphorylation, increased the expression of proIL-1 $\beta$ (Figure 5F).
Collectively, our data demonstrate that through its kinase activity PKR controls the induction of proteins to limit the assembly and ensuing activity of the cryopyrin inflammasome.

\section{Discussion}

With this report, four studies have now assessed the 
A

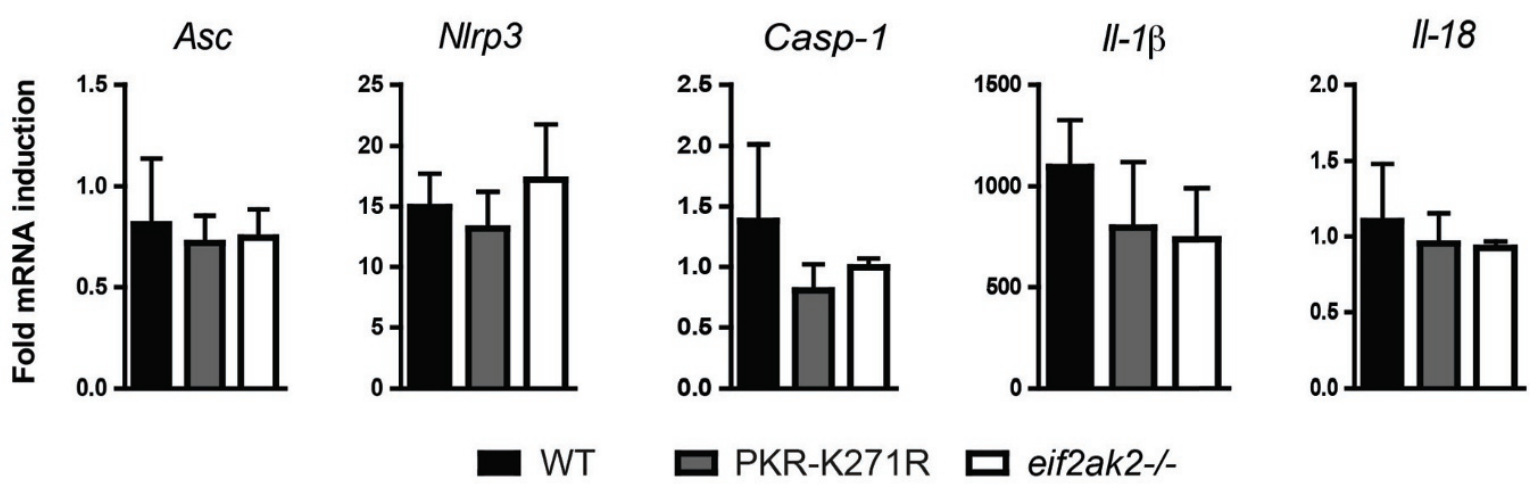

B

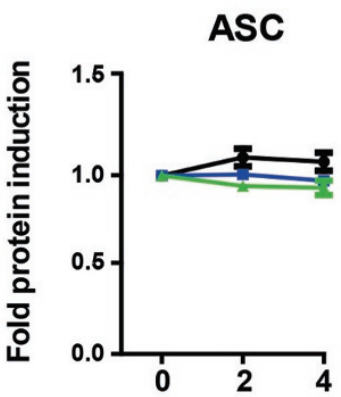

NLRP3

Pro-CASP-1

Pro-IL-1 $\beta$
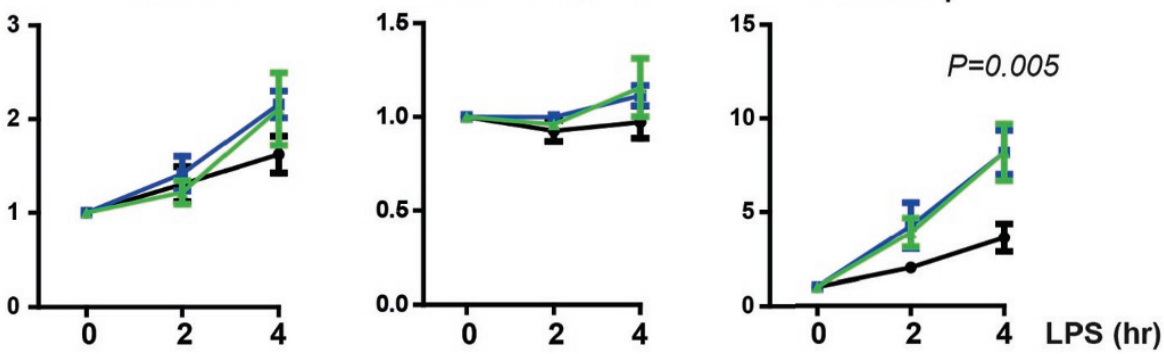

$\rightarrow$ WT $\quad-$ PKR-K271R $\quad$ - eif2ak2-/-

C

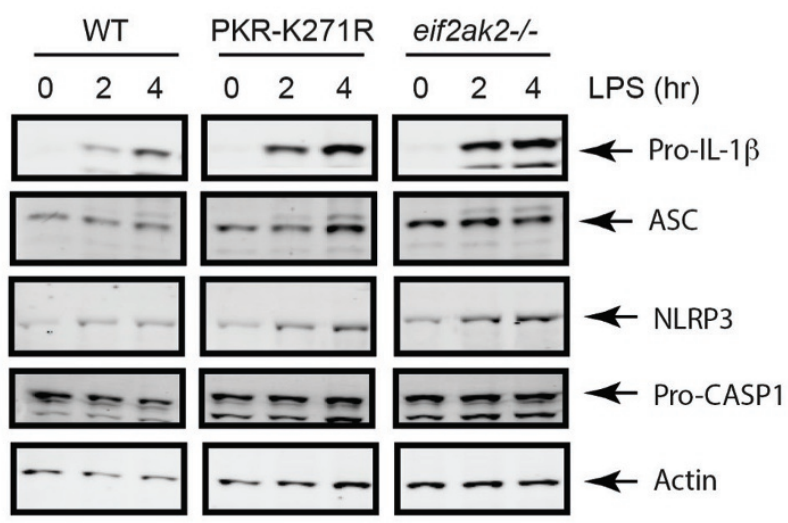

$\mathrm{E}$

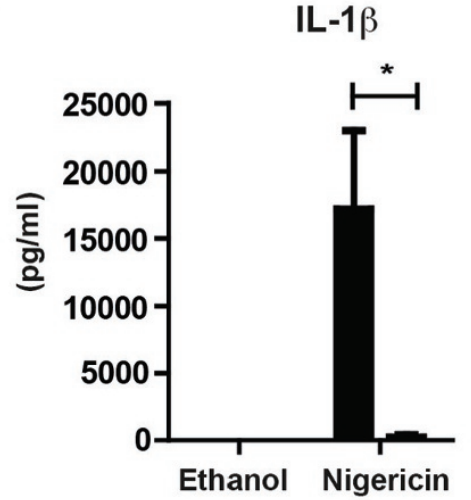

IL-18

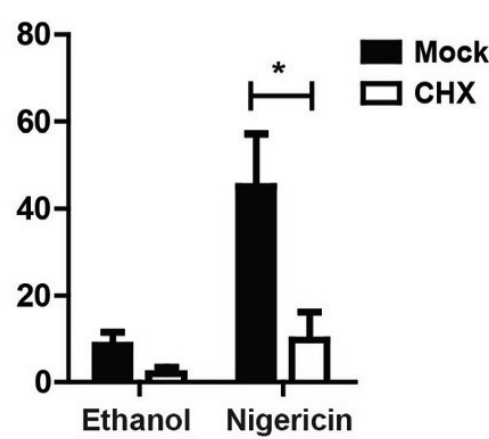

D

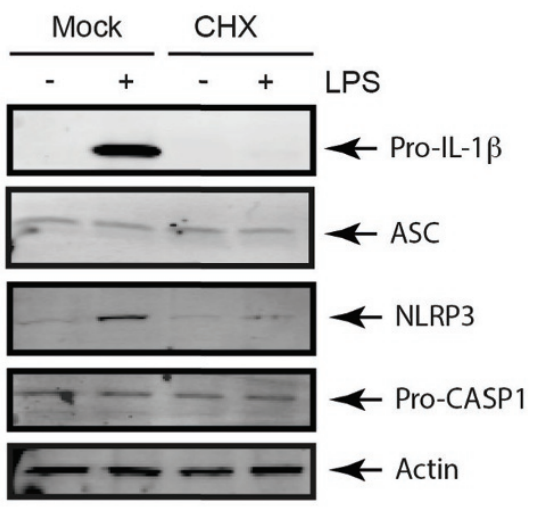

$\mathrm{F}$

Pro-IL-1 $\beta$

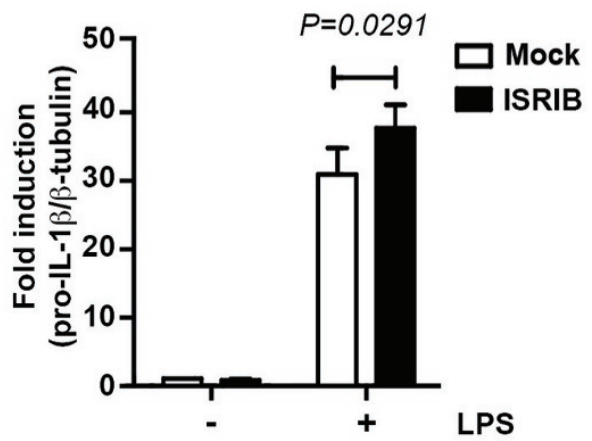


role of PKR in inflammasome activation $[3,5,6]$. There is disagreement between each, with Lu et al. [3] and Hett et al. [6] proposing that PKR promotes inflammasome activity, although there is a discrepancy over the dependence for kinase activity between these reports. In contradicting to this, $\mathrm{He}$ et al. [5] concluded that there was no role for PKR in controlling the inflammasomes, and we show that PKR represses inflammasome activity. We discuss differences between each study here that account for these discrepancies.

The report by He et al. [5] most closely approximates our findings. Their study assessed inflammasome activity in bone marrow-derived macrophages (BMDMs) isolated from both PKR-ablated mouse strains that were used by either $\mathrm{Lu}$ et al. [3] or ourselves that targeted either exons 12 [22] or 2-3 [15] of the eIF2 $\alpha k 2$ locus, respectively. Although these researchers reported no role for PKR, we contend that close examination of their data from the exon-12-targeted mouse shows that it supports our findings. This data shows a modest increase in pro-CASP-1, -IL$1 \beta$ and $-\mathrm{IL}-18$ processing, and a corresponding elevation of IL-1 $\beta$ levels in response to at least one of the stimuli used in the PKR-ablated cells. The cytokine levels are more significant than immediately apparent, if considered in relation to the accompanying measures of TNF $\alpha$ in wild-type and PKR-ablated cells. Against this, the data generated from the exon-2-3-targeted transgenic mouse showed no role for PKR. However, these comparisons were made between homozygous and heterozygous mutant cells, which risks diminishing the effect of PKR through a gene dosage effect. Consequently, we contend that the report by He et al. [5] generally accords with our findings.

Also in agreement with $\mathrm{He}$ et al. [5], we did not observe PKR dependence for all stimuli that activate the inflammasome and propose that this is, at least in part, due to indirect effects of PKR on the cell [17]. Effects of PKR on the cell cytoskeleton mean that the uptake of some stimuli is not equivalent between wild-type and
PKR-ablated cells, and this activation of the inflammasomes is not equivalent. For this reason, we present data in which the inflammasome was solely activated by treatment with nigericin.

In addition, we posit that our findings and those of He et al. [5] would be more analogous if conducted in equivalent cell models. He et al. [5] performed their experiments in BMDM. In our hands, BMDM demonstrate diminished PKR-dependent responses (Supplementary information, Figure S1). BMDM requires cytokine modulation for culture and survival. We contend that PKR-dependent responses are best measured in cells that are differentiated in vivo and therefore used primary macrophages isolated without manipulation. Related to this, the cell model used in the related report by Hett et al. [6] is even less favorable. This study tested PKR control of inflammasome activity in immortalized murine cell lines. We are able to replicate the phenomenon reported by Hett et al. [6] using the human monocytic cell line THP-1, by knocking down PKR expression by RNA interference. Accordingly, we do not contest their findings in this experimental context, but contend that such immortalized monocytic cell lines do not recapitulate PKR-dependent cell signaling. Apposite to this, the established function of PKR to control protein translation is best evidenced in our experiments performed in primary peritoneal macrophages.

It has been suggested that discordant responses that have previously been recorded between the differently targeted PKR transgenic animals [23, 24], could be the consequence of residual peptides that may be expressed in each animal. However, the demonstration by He et al. [5] of the relative equivalence of the response in BMDM isolated from either mouse, argues against this explanation. Also, our demonstration of equivalent responses in macrophages isolated from kinase-dead and PKR-ablated mice further repudiates this explanation.

A potentially significant point of variance between the studies is the murine strains that were used. Our re-

Figure 5 PKR represses translation of inflammasome constituents. (A) Induction of the indicated mRNAs, measured by quantitative PCR, in peritoneal macrophages isolated from WT and PKR mutant mice $(n=3-4)$, treated for $1 \mathrm{~h}$ with LPS (10 $\mathrm{ng} / \mathrm{ml})$. (B, C) Quantitation of the indicated proteins (B), measured by immune blot (C) using anti-ASC, -NLRP3, -CASP-1 and -IL-1 $\beta$ antibodies in peritoneal macrophages from WT and PKR mutant mice $(n=4)$, treated for the indicated times with LPS $(10$ $\mathrm{ng} / \mathrm{ml}$ ). Primary antibodies were detected with fluorescent secondary antibodies then quantitated and normalized to the levels of actin or, alternatively, ASC (equivalent to the actin normalization shown). (D) Detection of the indicated proteins by immune blot with anti-ASC, -NLRP3, -CASP-1 and -IL-1 $\beta$ antibodies in peritoneal macrophages from WT mice treated with cycloheximide $(\mathrm{CHX}, 1 \mu \mathrm{g} / \mathrm{ml})$ for $1 \mathrm{~h}$, then LPS $(10 \mathrm{ng} / \mathrm{ml})$ for $4 \mathrm{~h}$. (E) Production of IL-1 $\beta$ and IL-18 in the culture supernatant of peritoneal macrophages from WT mice treated with cycloheximide $(1 \mu \mathrm{g} / \mathrm{ml})$ for $1 \mathrm{~h}$, then LPS $(10 \mathrm{ng} / \mathrm{ml})$ for $4 \mathrm{~h}$ followed by nigericin $(10 \mu \mathrm{M})$ for $30 \mathrm{~min}$, as measured by ELISA $(n=3)\left({ }^{*} P<0.05\right)$. (F) Detection of the expression of pro-IL-1 $\beta$ by immune blot using anti$\mathrm{IL}-1 \beta$ antibodies in peritoneal macrophages from WT mice treated with ISRIB (10 nM) for $1 \mathrm{~h}$, then LPS (10 ng/ml) for $4 \mathrm{~h}$. 
port tested inflammasome activity in cells isolated from isogenic C57B16 mice, while the other reports used animals that were variable on the $129 \mathrm{v}$ background. This could be significant, as the 129 strain has attenuated inflammasome activity due to diminished CASP-11 expression [25]. As expression of CASP-11 is induced by the CCAAT-enhancer-binding protein homologous protein, the expression of which is controlled by eIF $2 \alpha$ phosphorylation [26], PKR activity could rescue the impairment in the 129 strain by restoring CASP-11 expression. Lu et al. [3] used macrophages isolated from a mixed 129Sv/ BALBc background. Although they also established the $e I F 2 \alpha k^{-1-}$ on a C57BL/6 background, the activity of the inflammasome was not explicitly examined in cells from this strain. Moreover, their interpretation of the significance of IL- $1 \beta$ production in this C57B16J mouse infected with $E$. coli was complicated by diminished clearance, and thus, elevated bacterial burden in the wild-type compared with $e I F 2 \alpha k 2^{-/}$mice. This result appears antithetic to the proposed proinflammatory role for PKR. The data generated by He et al. [5] were from animals that were not sufficiently backcrossed with a CASP-11 competent strain to definitively remedy this defect. However, the exon-12-targeted animal, which we contend shows a degree of PKR-dependent inhibition of inflammasome activity, had been backcrossed with the BALBc strain for six generations. Contrary to this speculation, a report by Kayagake et al. [25] does not favor a role for CASP-11 in nigericin-induced inflammasome activation. This independence from CASP-11 relies upon the exclusion of the priming stimulus, LPS, from the cytosol for exclusive signaling via Toll-like receptor 4 [27, 28], as LPS directly binds to the CARD domain of CASP-11 [28]. However, release of LPS into the cell cytoplasm and activation of CASP-11 are dependent on interferon signaling [29, 30], which is promoted by PKR activity. Consequently, the requisite exclusion of LPS may not be equivalent between wild-type and PKR-ablated cells.

As to the mechanism by which PKR mediates these effects, Lu et al. [3] proposed that PKR functions as a scaffold molecule that directly interacts with protein components to promote assembly of the inflammasome. How PKR might promote the homotypic interactions between the reciprocal pyrin and caspase-recruitment domains of cryopyrin, ASC and CASP-1, is not clear. Insights into the assembly of the inflammasome gained from structural studies indicate that these interacting domains are sufficient to mediate nucleation, with assembly being initiated by the level and state of cryopyrin [31]. The data from He et al. [5] and ourselves do not support a role for PKR in inflammasome assembly. Our demonstration that PKR restricts the induction of the constitu- ents of the inflammasome offers an alternative explanation as to how PKR controls this response.

A principal function of PKR is to control translation initiation by phosphorylation of eIF $2 \alpha$. PKR-dependent control of gene expression most impacts proteins that are labile or that must be induced to exert their influence. This effect is best evidenced in the current context by the control of pro-IL-1 $\beta$ levels through the kinase activity of PKR. This effect was separately confirmed by using an inhibitor of the integrated stress response pathway that is induced by eIF2 $\alpha$ phosphorylation. However, control of pro-IL-1 $\beta$ expression alone does not account for reduced processing of this cytokine or the constitutively expressed IL-18, altered ASC nucleation and increased CASP-1 activation. The expression of cryopyrin was also reduced by PKR activity, although our measure of repression did not reach statistical significance. Repression of the induction of cryopyrin was confirmed with the protein translation inhibitor cycloheximide, which similarly repressed inflammasome activity. As cryopyrin is critical to the initial nucleation step in inflammasome activation [31], reduction of its levels could account for the diminished activity of the inflammasome. Such a mechanism would be in keeping with the proposition of how inflammasomes form and the recognition that a large proportion of patients with cryopyrin-associated periodic syndrome appear to suffer from the consequence of increased expression of the components of the inflammasome, rather than an innately more active inflammasome [32]. However, we have not established that the reduced levels of cryopyrin wholly account for the reduced activity of this inflammasome, and therefore, it cannot be excluded that PKR activity regulates the expression of alternative transcripts that control this response.

To summarize, our study directly contests the claim by $\mathrm{Lu}$ et al. [3] that PKR promotes the activity of the cryopyrin inflammasome. We assert that there is partial agreement between our findings and another related report by $\mathrm{He}$ et al. [5], with the remaining differences due to diminished PKR-dependent responses because of heterozygosity, separate stimuli and the different cell models used. The current understanding of how inflammasomes form, together with our data and that from $\mathrm{He}$ et al. [5], does not support the proposition that PKR promotes inflammasome assembly by acting as a scaffold molecule as proposed by Lu et al. [3]. Rather, our data predict that PKR diminishes inflammasome activity by controlling protein translation to repress the induction of factors that are critical for the activity of the cryopyrin inflammasome. These findings caution against the previously proposed therapeutic strategy to inhibit PKR to treat inflammation. 


\section{Materials and Methods}

Mice

All mice used in experiments were treated in accordance with practices approved by the Monash University, Monash Medical Centre animal ethics committee (Melbourne, Australia), as obligated by the Australian Code of Practice for the care and use of animals for scientific purposes, in compliance with standards mandated by the Australian National Health and Medical Research Council and National Institutes of Health. Mice were bred in high barrier-specific pathogen-free conditions on a C57BL/6 genetic background. Mice were sacrificed for cell collection between 7 and 10 weeks of age.

\section{Cells}

Primary peritoneal macrophages were harvested by injecting $10 \mathrm{ml}$ phosphate-buffered saline (PBS, Life Technologies, USA) into the peritoneal cavity of the mice. The cell suspension was withdrawn from the peritoneum and centrifuged at 1300 r.p.m. for $5 \mathrm{~min}$. The cell pellets were re-suspended in RPMI-1640 medium with $10 \%$ fetal bovine serum (FBS, Lonza, Switzerland) at $2 \times 10^{5}$ cells $/ \mathrm{ml}$ and plated on 24-well plates overnight. The media were removed. Cells were then washed with PBS and replenished with RPMI-1640 medium with $1 \%$ FBS. After $2 \mathrm{~h}$, cells were primed with $10 \mathrm{ng} / \mathrm{ml}$ LPS for $4 \mathrm{~h}$ before addition of inflammasome activators.

\section{Reagents}

2-Aminopurine (2-AP) was purchased from GE Healthcare Life Sciences. Anti-ASC (AL177) and -NLRP3 antibodies (Cryo2 ) were purchased from Adipogen (USA). The anti-IL-1 $\beta$ antibody (ab9722) was purchased from Abcam (UK). Anti-Caspase-1 p10 (M20), -PKR (sc-6282) and -eIF2 $\alpha$ (sc-81261) antibodies were from Santa Cruz Biotechnology, Inc. (USA). The anti-pS ${ }^{51}$-eIF2 $\alpha$ (447289) was from Invitrogen. Nigericin was purchased from Invivogen (USA). LPS was extracted from Escherichia coli K-235 (Cat. No: L2143); (Sigma-Aldrich, USA). ISRIB was purchased from Sigma-Aldrich (Cat. No: SML0843). FLICA 660 Caspase-1 (Cat No: 9122) was purchased from Immunochemistry Technologies, LCC (MN, USA) and used as recommended by the manufacturer. PolyI:C (GE Healthcare Life Sciences) was transfected into cells with JetPRIME (Polyplus Transfection).

\section{Assessing ASC speck formation and CASP-1 activity}

Cells were primed with LPS for $4 \mathrm{~h}$, and then treated with nigericin for $15 \mathrm{~min}$. Cells were fixed by $10 \%$ formalin and permeabilized by $0.1 \%$ Triton X-100 in PBS, then stained with FLICA 660 Caspase-1 (according to the manufacturer's protocol) or stained with anti-ASC antibody, followed by incubation with secondary antibodies conjugated with fluorophore NL557 (R\&D Systems). Cell nuclei were stained with Hoechst 33342 (Life Technologies, USA). Data were captured as Z-stacks of $12.8 \mu \mathrm{m}$ thickness at $0.2 \mu \mathrm{m}$ intervals from five fields of each treatment by the DeltaVision microscope. Micrographs containing 4-8 cells per field were analyzed by Imaris software using "Cell” analysis. ASC specks and activated CASP-1 were defined as vesicles with a diameter of $1 \mu \mathrm{m}$ and $0.5 \mu \mathrm{m}$, respectively.

\section{LDH assay}

Cell culture media were collected and analyzed by LDH Cytotoxicity Assay (Cayman Chemicals, USA) according to the manufacturer's instructions. The data were expressed as fold induction as compared with the mock-treated cells.

\section{ELISA}

The levels of IL-1 and IL-18 in the cell culture media were measured by ELISA kits from BD Biosciences (USA) and MBL (USA), respectively, according to the manufacturer's protocols.

\section{Immune blot procedure}

Cell lysates were harvested by RIPA buffer $(10 \mathrm{mM}$ Tris-HCl, $\mathrm{pH}$ 8.0, $140 \mathrm{mM} \mathrm{NaCl}, 1 \mathrm{mM}$ EDTA, $0.5 \mathrm{mM}$ EGTA, 1\% Triton $\mathrm{X}-100,0.1 \%$ sodium deoxycholate, $0.1 \%$ sodium dodecyl sulphate (SDS), protease inhibitor cocktail (P2714, Sigma-Aldrich, USA), $200 \mathrm{nM}$ sodium orthovanadate, $50 \mathrm{mM} \mathrm{NaF}$ ). To validate that the observed altered electrophoretic mobility of wild-type PKR compared with kinase-dead PKR was due to autophosphorylation, we incubated whole-cell lysates with the Lambda protein phosphatase as per the manufacturer's instructions (NEB). Proteins were heat denatured in sample buffer (125 mM Tris- $\mathrm{HCl}, \mathrm{pH} 6.8,4 \%$ SDS, 20\% glycerol, 5\% $\beta$-mercaptoethanol, $0.01 \%$ bromophenol blue), separated by $8 \%-15 \%$ SDS-PAGE and transferred to Immobilon-FL Membrane (Millipore, USA). These membranes were probed with different primary antibodies and fluorophore-conjugated secondary antibodies for visualizing the proteins by Odyssey Imaging System (LI-COR, USA). Protein bands were quantified by Odyssey Imaging Software (LI-COR, USA).

\section{Quantitative real-time $P C R$}

Quantitative PCR (q-PCR) was performed in triplicate on an Applied Biosystems 7700 Prism real-time PCR machine using SYBR Green-based (Applied Biosystems, USA) PCR with the following primers: Pycard 5'-CAGAGTACAGCCAGAACAGGACAC-3', 5'-GTGGTCTCTGCACGAACTGCCTG-3'; Nlrp3 5'-CGAGACCTCTGGGAAAAAGCT-3', 5'-GCATACCATAGAGGAATGTGATGTACA-3'; Casp1 5'-TCCGCGGTTGAATCCTTTTCAGA-3', 5'-ACCACAATTGCTGTGTGTGCGCA-3; $I l-1 \beta$ 5'-TCTGGGATCCTCTCCAGCCAAG-3', 5'-TCAGGACAGCCCAGGTCAAAGG-3'; Il-18 5'-TTGGCCCAGGAACAATGGCTGC-3', 5'-TGCGGTTGTACAGTGAAGTCGG-3'. Expression of mRNA was assessed after normalization of each target to the $18 \mathrm{~S}$ ribosomal RNA amplicon (detected with the primers 5'-AGTCCCTGCCCTTTGTACACA-3', 5'-CGATCCGAGGGCCTCACTA-3') by the change in cycling threshold $\left(\Delta \mathrm{C}_{\mathrm{T}}\right)$ method and calculated based on $2^{-\Delta \mathrm{CT}}$. Results are expressed as relative gene expression for each target gene.

\section{Statistical analysis}

All statistical analyses were performed with GraphPad Prism software. Differences were calculated using the unpaired, twotailed $t$-test for pairwise comparisons, or by linear regression calculated for each genotype, for the temporal data. $P$-values $<0.05$ were considered significant.

\section{Acknowledgments}

We thank Dr Frances Cribbin for assistance with preparation of the manuscript, and Drs Camden Lo and Kirstin Elgass for advice 
on fluorescence microscopy. This work was supported by a grant from the National Health and Medical Research Council (NHMRC) of Australia (Grantor ID: 1043398), an NHMRC Early Career Fellowship to HCH Yim (1052730), a Ron Evans Cancer Research Fellowship (to HCH Yim) and the Victorian Government's Operational Infrastructure Support Program.

\section{Author Contributions}

HCHY and AJS designed the study; HCHY, DW and LY performed the experiments; CLW and PWF generated the K271RPKR mice; HCHY, BRGW and AJS analyzed the data; AJS wrote the paper.

\section{Competing Financial Interests}

The authors declare no competing financial interests.

\section{References}

1 Harding HP, Novoa I, Zhang Y, et al. Regulated translation initiation controls stress-induced gene expression in mammalian cells. Mol Cell 2000; 6:1099-1108.

2 Harding HP, Zhang Y, Zeng $\mathrm{H}$, et al. An integrated stress response regulates amino acid metabolism and resistance to oxidative stress. Mol Cell 2003; 11:619-633.

3 Lu B, Nakamura T, Inouye K, et al. Novel role of PKR in inflammasome activation and HMGB1 release. Nature 2012; 488:670-674.

4 Guarda G, Braun M, Staehli F, et al. Type I interferon inhibits interleukin-1 production and inflammasome activation. Immunity 2011; 34:213-223.

5 He Y, Franchi L, Nunez G. The protein kinase PKR is critical for LPS-induced iNOS production but dispensable for inflammasome activation in macrophages. Eur J Immunol 2013; 43:1147-1152.

6 Hett EC, Slater LH, Mark KG, et al. Chemical genetics reveals a kinase-independent role for protein kinase $\mathrm{R}$ in pyroptosis. Nat Chem Biol 2013; 9:398-405.

7 Horng T, Barton GM, Medzhitov R. TIRAP: an adapter molecule in the Toll signaling pathway. Nat Immunol 2001; 2:835841.

8 Yang YL, Reis LF, Pavlovic J, et al. Deficient signaling in mice devoid of double-stranded RNA-dependent protein kinase. EMBO J 1995; 14:6095-6106.

9 Bonnet MC, Weil R, Dam E, Hovanessian AG, Meurs EF. PKR stimulates NF-kappaB irrespective of its kinase function by interacting with the IkappaB kinase complex. Mol Cell Biol 2000; 20:4532-4542.

10 Bonnet MC, Daurat C, Ottone C, Meurs EF. The N-terminus of PKR is responsible for the activation of the NF-kappaB signaling pathway by interacting with the IKK complex. Cell Signal 2006; 18:1865-1875.

11 Gil J, Garcia MA, Gomez-Puertas P, et al. TRAF family proteins link PKR with NF-kappa B activation. Mol Cell Biol 2004; 24:4502-4512.

12 Vince JE, Wong WW, Gentle I, et al. Inhibitor of apoptosis proteins limit RIP3 kinase-dependent interleukin-1 activation. Immunity 2012; 36:215-227.

13 Hanks SK, Quinn AM. Protein kinase catalytic domain sequence database: identification of conserved features of pri- mary structure and classification of family members. Methods Enzymol 1991; 200:38-62.

14 Katze MG, Wambach M, Wong ML, et al. Functional expression and RNA binding analysis of the interferon-induced, double-stranded RNA-activated, 68,000-Mr protein kinase in a cell-free system. Mol Cell Biol 1991; 11:5497-5505.

15 Chong KL, Feng L, Schappert K, et al. Human p68 kinase exhibits growth suppression in yeast and homology to the translational regulator GCN2. EMBO J 1992; 11:1553-1562.

16 Sadler AJ, Williams BR. Interferon-inducible antiviral effectors. Nat Rev Immunol 2008; 8:559-568.

17 Irving AT, Wang D, Vasilevski O, et al. Regulation of actin dynamics by protein kinase $\mathrm{R}$ control of gelsolin enforces basal innate immune defense. Immunity 2012; 36:795-806.

18 Perregaux D, Barberia J, Lanzetti AJ, Geoghegan KF, Carty TJ, Gabel CA. IL-1 beta maturation: evidence that mature cytokine formation can be induced specifically by nigericin. $J$ Immunol 1992; 149:1294-1303.

19 Lee SB, Esteban M. The interferon-induced double-stranded RNA-activated protein kinase induces apoptosis. Virology 1994; 199:491-496.

20 Hsu LC, Park JM, Zhang K, et al. The protein kinase PKR is required for macrophage apoptosis after activation of Toll-like receptor 4. Nature 2004; 428:341-345.

21 Barber GN, Wambach M, Wong ML, Dever TE, Hinnebusch AG, Katze MG. Translational regulation by the interferon-induced double-stranded-RNA-activated 68-kDa protein kinase. Proc Natl Acad Sci USA 1993; 90:4621-4625.

22 Abraham N, Stojdl DF, Duncan PI, et al. Characterization of transgenic mice with targeted disruption of the catalytic domain of the double-stranded RNA-dependent protein kinase, PKR. J Biol Chem 1999; 274:5953-5962.

23 Cao SS, Song B, Kaufman RJ. PKR protects colonic epithelium against colitis through the unfolded protein response and prosurvival signaling. Inflamm Bowel Dis 2012; 18:17351742.

24 Rath E, Berger E, Messlik A, et al. Induction of dsRNA-activated protein kinase links mitochondrial unfolded protein response to the pathogenesis of intestinal inflammation. Gut 2012; 61:1269-1278.

25 Kayagaki N, Warming S, Lamkanfi M, et al. Non-canonical inflammasome activation targets caspase-11. Nature 2011; 479:117-121.

26 Endo M, Mori M, Akira S, Gotoh T. C/EBP homologous protein (CHOP) is crucial for the induction of caspase-11 and the pathogenesis of lipopolysaccharide-induced inflammation. $J$ Immunol 2006; 176:6245-6253.

27 Kayagaki N, Wong MT, Stowe IB, et al. Noncanonical inflammasome activation by intracellular LPS independent of TLR4. Science 2013; 341:1246-1249.

28 Shi J, Zhao Y, Wang Y, et al. Inflammatory caspases are innate immune receptors for intracellular LPS. Nature 2014; 514:187-192.

29 Meunier E, Dick MS, Dreier RF, et al. Caspase-11 activation requires lysis of pathogen-containing vacuoles by IFN-induced GTPases. Nature 2014; 509:366-370.

30 Rathinam VA, Vanaja SK, Waggoner L, et al. TRIF licenses caspase-11-dependent NLRP3 inflammasome activation by gram-negative bacteria. Cell 2012; 150:606-619. 
31 Lu A, Magupalli VG, Ruan J, et al. Unified polymerization mechanism for the assembly of ASC-dependent inflammasomes. Cell 2014; 156:1193-1206.

32 Anderson JP, Mueller JL, Misaghi A, et al. Initial description of the human NLRP3 promoter. Genes Immun 2008; 9:721726.

(Supplementary information is linked to the online version of the paper on the Cell Research website) 